\title{
Dermatite de contato agravada por outra sensibilidade em automedicação
}

\author{
Paulo Eduardo Silva Belluco ${ }^{1}$ (D), Fabíola da Silva Maciel Azevedo² (D), Cejana Rebouças Fernandes de Lima $^{3}$ (D), \\ Rosana Zabulon Feijó Belluco ${ }^{1}\left(\mathbb{D}\right.$, Carmelia Matos Santiago Reis ${ }^{1}[\mathbb{C}$
}

\begin{abstract}
RESUMO
Dermatite alérgica de contato é uma doença cutânea inflamatória, não infecciosa, cuja base do tratamento é a identificação e eliminação do agente causal. Cocoamidopropil betaína é um surfactante muito utilizado nos produtos de uso pessoal, notadamente de uso capilar. Essa substância não está presente na bateria padrão brasileira. Neomicina é um antibiótico usado em preparações tópicas. Objetivamos mostrar paciente que desenvolveu alergia no couro cabeludo e que fez erroneamente automedicação com produto que continha substância a qual era ainda mais sensível. O caso é de uma mulher, 36 anos, evoluindo há dois meses com eczema pruriginoso, em áreas de implantação capilar e nuca. Relatava progressiva piora. Diante da suspeita de dermatite de contato, foi realizado teste de contato, utilizando-se da bateria padrão Latino-Americana. Com 96 horas (D4) evidenciou-se positividade leve $(+)$ para cocoamidopropil betaína e forte $(++)$ para neomicina. O resultado positivo para a neomicina foi intrigante, uma vez que a observação do rótulo dos produtos de uso pessoal não a continha. Diante do resultado do teste, após ser questionada novamente, ela confirmou a omissão da automedicação diária com pomada de neomicina. Em conclusão, mostramos a alergia a produtos de uso capilar. Reforçamos a necessidade de se fazer um teste de contato com bateria padrão atualizada. Por fim, alertamos sobre o risco da automedicação.
\end{abstract}

Palavras-chave: Dermatite alérgica de contato, Cocoamidopropil betaína, Neomicina, Teste de contato.

1. Escola Superior de Ciências da Saúde - ESCS - Brasília / DF

2. Hospital de Força Aérea de Brasília - Brasília / DF

3. Hospital Regional da Asa Norte - HRAN - Brasília / DF 


\section{INTRODUÇÃO}

A Dermatite de contato é patologia cutânea comum, afetando em média 19,5\% da população e impõe uma significativa carga de sofrimento humano ${ }^{1}$. Talvez esse seja um dos motivos que fazem os pacientes procurarem avidamente a sua solução através de um medicamento salvador, abusando de automedicações. A doença é largamente prevenível ${ }^{1}$, assim, o melhor tratamento passa pela identificação do agente causal e sua eliminação. $O$ tratamento sintomático é necessário, mas especialmente através de medicamentos tópicos anti-inflamatórios prioritariamente (corticoides tópicos), pois a doença é efetivamente uma doença caracterizada por uma reação de hipersensibilidade retardada inflamatória. Objetivamos mostrar paciente com eczema a cocoamidopropil betaína, componente comum de xampus e condicionadores, que se automedica com pomada a base de neomicina, a qual apresenta sensibilidade ainda maior. O resultado é a piora progressiva. Este estudo foi desenvolvido de acordo com a Resolução 466/12 do Conselho Nacional de Saúde e foi aprovada pelo Comitê de Ética em Pesquisa da Fundação de Ensino e Pesquisa em Ciências da Saúde com o número CAAE 22295219.9.0000.5553.

\section{RELATO DE CASO}

Relatamos o caso de uma paciente do sexo feminino, 36 anos, bancária, evoluindo há dois meses com quadro de lesões com placas eritematosas, erosadas, escoriadas e descamativas em couro cabeludo, região cervical bilateralmente (Figura 1), nuca (Figura 2) e na implantação capilar (Figura 3) em têmporas e algumas delas com aspecto de liquenificação. Ela referia que apresentava um prurido intenso e que notava progressiva piora com o tempo, levando-a a procurar o prontosocorro que a referiu a nosso serviço especializado. Negava antecedentes de alergia, uso de medicamentos ou realização de procedimentos cosméticos, exceto a pintura do cabelo, de coloração escura.

Feito o diagnóstico clínico presuntivo, a paciente foi submetida ao teste de contato, sendo utilizado a bateria padrão Latino-Americana contendo 40 substâncias. Para isso foram utilizados quatro contensores - fitas adesivas hipoalergênicas Alergochamber ${ }^{\circledR}$ (Neoflex Biotecnologia Ltda ${ }^{\circledR}$ ) previamente preparados com dez substâncias cada. As substâncias do teste foram manipuladas, seguindo o seu "CAS number", pela IPI ASAC BRASIL ${ }^{\circledR}$,

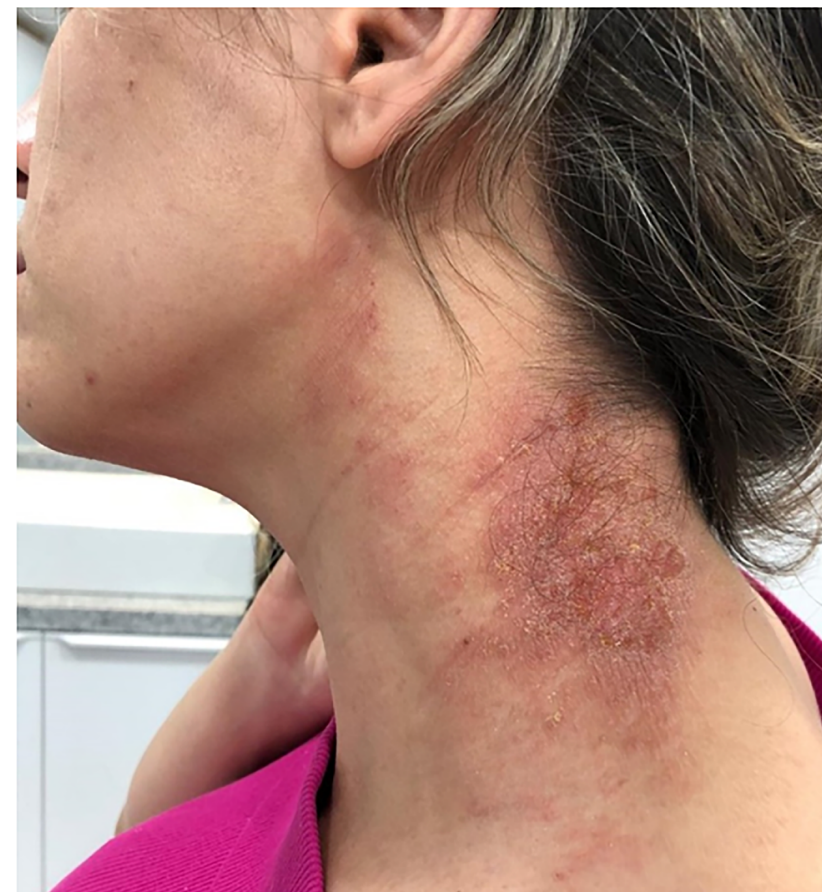

Figura 1. Eczema com erosões e fissuras na face lateral do pescoço.

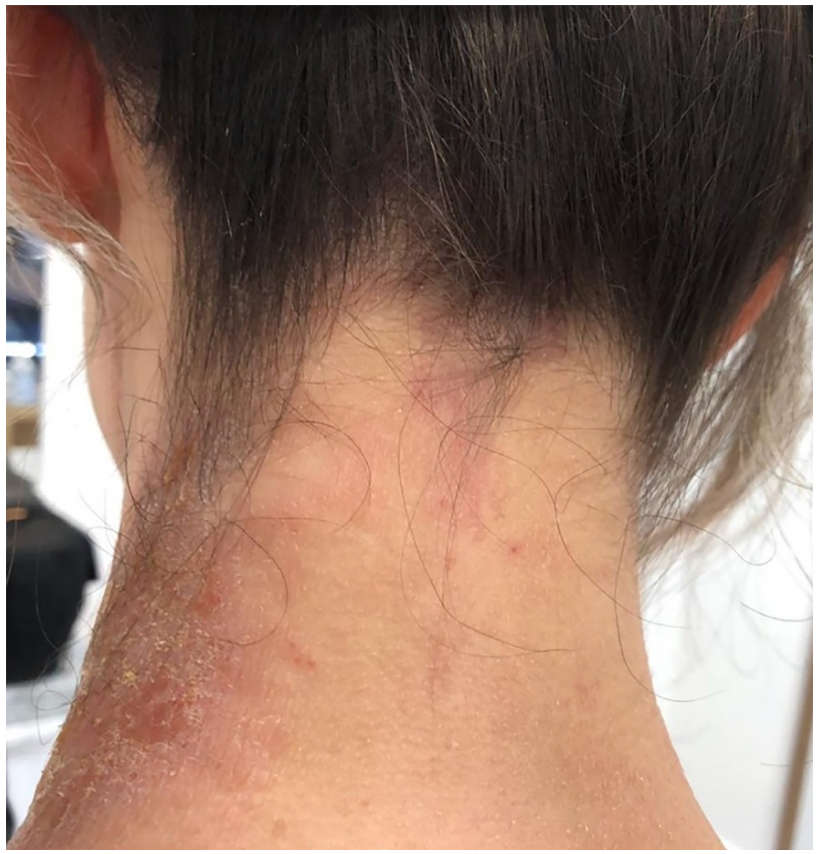

Figura 2. Placa eritematosa na nuca.

conforme orientação do Colégio Ibero LatinoAmericano de Dermatologia2. Foram realizadas leituras com 48 (D2) e 96 horas (D4). Na segunda leitura, foram observadas reação positiva leve $(+)$ a cocoamidopropil betaína a $1 \%$ em veículo aquoso e forte $(++)$ a neomicina a $20 \%$ em vaselina (Figura 4 ). 


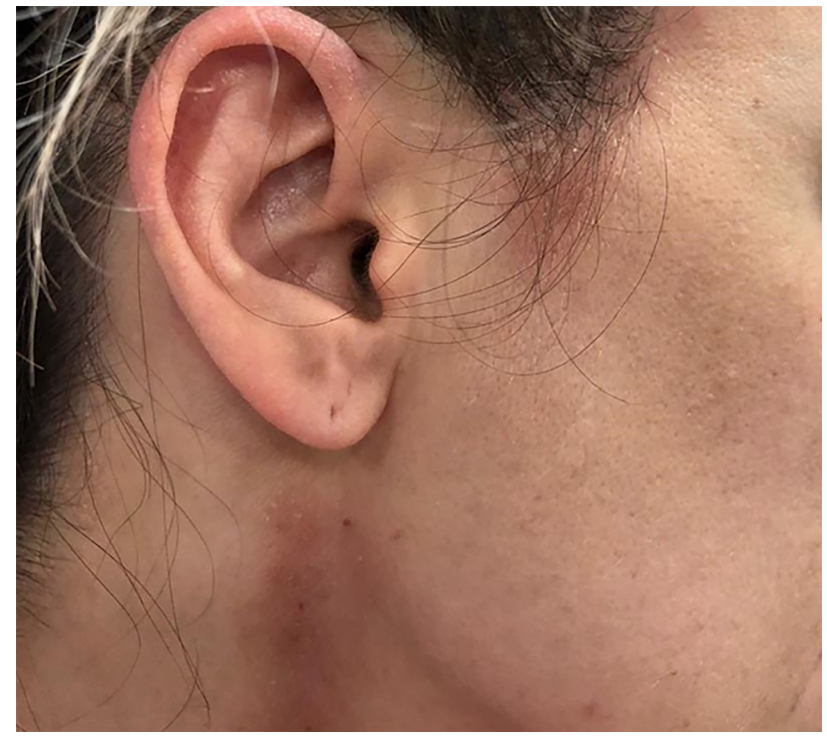

Figura 3. Mácula na área de implantação do cabelo.

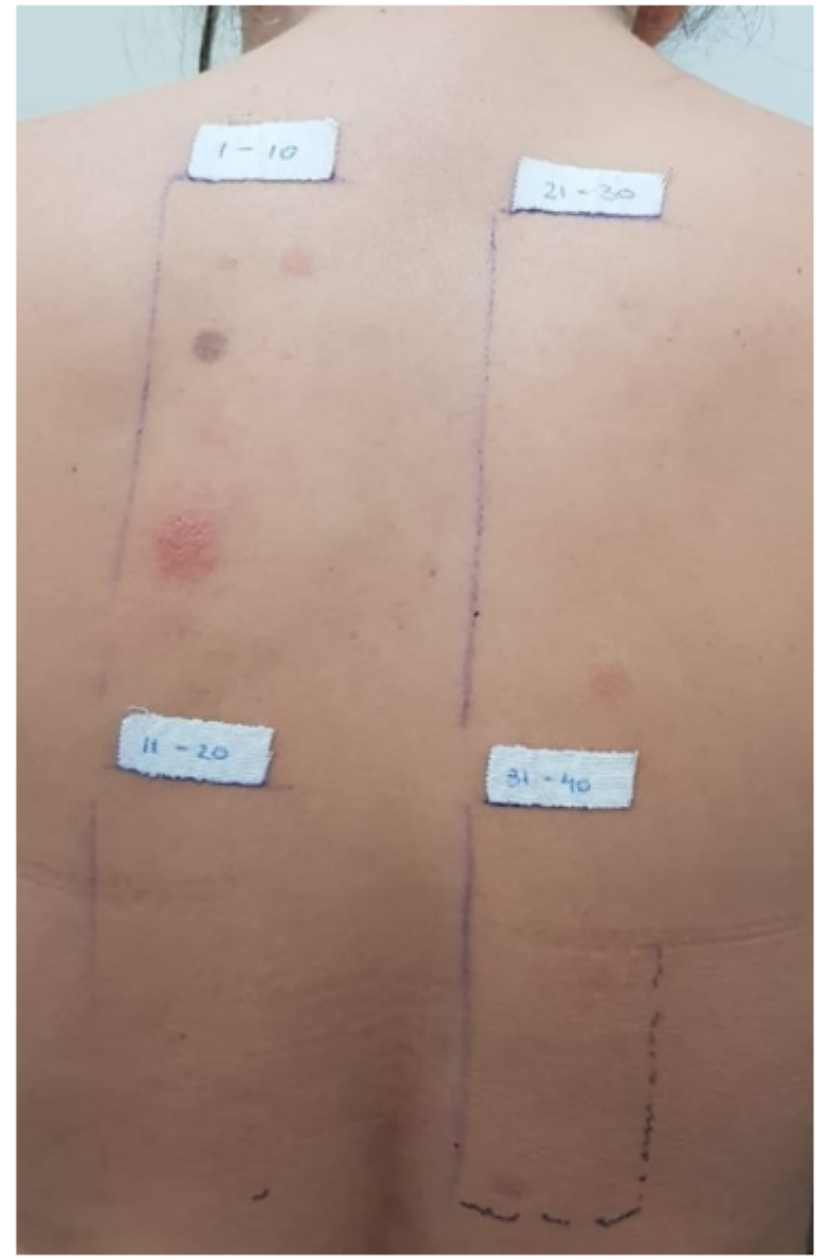

Figura 4. Leitura em D4 - Neomicina ++ e cocoamidopropil betaína +.

\section{DISCUSSÃO}

Dermatite de contato é uma doença cutânea inflamatória comum, não infecciosa, que ocorre pelo contato direto ou indireto com substâncias que são prejudiciais à pele. Tem como uma de suas características principais o fato de ser largamente prevenível ${ }^{1}$. Assim, a base do tratamento é a identificação e a eliminação do fator gerador do eczema. Relatamos o caso de uma paciente que, ao invés de procurar identificar o agente causal, seja empiricamente evitando produtos suspeitos, seja comprovadamente através de um teste de contato, preferiu se automedicar. A automedicação, além de não ser indicada por si só, foi realizada com um antimicrobiano. Obviamente que a doença não sendo infecciosa, não apresentaria melhora. E mais, apresentou clinicamente uma nítida progressão do eczema justamente por ser alérgica ao produto que estava utilizando especificamente para o tratamento.

Devemos ressaltar que a despeito do fato de que o couro cabeludo é constantemente exposto a vários alérgenos diariamente, a dermatite alérgica de contato é incomum nesse local ${ }^{3}$. Porém, na avaliação clínica da paciente, nitidamente observamos o acometimento no padrão conhecido como rinse-off de produtos de uso capilar. Nesse padrão há placas eczematosas nos lados da face e pescoço. As lesões são produzidas pelo alérgeno escorrendo ao longo da face. É típico de xampus, condicionadores e outros produtos que são aplicados temporariamente no couro cabeludo e fazem breve, mas contato recorrente com a pele da face $^{3}$. Caso tivesse procurado um especialista, essa observação clínica já teria sido feita, mudando-se os produtos em uso até antes de se fazer um teste de contato definitivo. Publicação recente, reforça que células $T$ regulatórias se concentram nos folículos pilosos, agindo em condições normais, promovendo tolerância a organismos residentes. Esse fato ajuda a explicar porque os eczemas não ocorrem comumente nessa região corporal ${ }^{4}$.

Cocoamidopropil betaína é um detergente sintético que tem sido progressivamente mais usado em cosméticos e produtos de higiene pessoal (por exemplo, xampus, soluções para lentes de contato, pasta de dentes, removedores de maquiagem, sabonetes, etc.) porque ele induz relativamente pouca irritação cutânea. No entanto, reação de hipersensibilidade retardada tem sido relatada e prevalência de sensibilidade de contato é estimada 
de 3 a 7,2\%. 0 índice crescente de sensibilização levou a cocoamidopropil betaína a ser considerada o "Alérgeno do Ano" em 20045. É fato que o teste do caso foi levemente positivo $(+)$. Nesses casos, reações positivas ao teste de contato devem ser cuidadosamente avaliadas para se julgar sua relevância clínica, em termos se há alguma relação entre a reação positiva e a dermatite encontrada ${ }^{6}$. Observamos claramente que a relevância estava presente, pois todos os xampus e condicionadores trazidos pela paciente para verificação de rótulos continham essa substância. Ou seja, o teste estava relacionado aos sintomas clínicos que levaram a paciente a buscar atendimento dermatológico (ela sofreu exposição ao alérgeno que resultou positivo no teste, sendo corresponsável pela dermatite atual) ${ }^{6}$.

Todos os pacientes com suspeita de dermatite alérgica de contato devem ser submetidos a teste com bateria padrão 7 . Se um alérgeno é achado na frequência acima de $1 \%$ na população, isso é considerado um percentual que justifica a inclusão da substância na série padrão ${ }^{8}$. A bateria padrão brasileira proposta em 1996 pelo Grupo Brasileiro de Dermatite de Contato, cujo estudo que a balizou foi publicado há 20 anos, não contempla a cocoamidopropil betaína9. Não encontramos estudos populacionais para mostrar a prevalência da sensibilidade a essa substância em nossa população. Somente o uso de uma bateria ampliada e atualizada como a bateria Latino-Americana proposta pelo Colégio Latino-Americano de Dermatologia em 2015, que possibilitou o diagnóstico da sensibilidade primária ${ }^{2}$. Nos Estados Unidos, a cocoamidopropil betaína está incluída na bateria padrão, tendo uma prevalência de positividade $1,6 \%$ na população testada ${ }^{10}$.

Neomicina é um antibiótico aminoglicosídeo comumente usado em medicações tópicas, tais como cremes e pomadas, assim como em colírios e gotas otológicas. Dermatite de contato relacionado a ela é comum em pacientes com dermatite em membros inferiores, dermatite atópica, eczema genital, e inflamação crônica ocular ou de ouvidos. A incidência da alergia a neomicina está por volta de $3,6 \%$ em populações submetidas a teste de contato e $1 \%$ em indivíduos sadios $^{11}$. Ela não se encontrava em nenhum dos produtos cosméticos trazidos pela paciente. Foi utilizada como automedicação erroneamente. Além de não resolver o eczema, acarretou numa progressiva piora levando a eczema grave e prurido incontrolável. Ressaltamos que na anamnese inicial, a paciente não revelou o seu uso. Somente após mostrarmos a intensidade da reação no seu dorso é que confessou que usava diariamente o produto contendo neomicina e que sentia a piora crescente. Na prática de realizarmos testes de contato há anos, observamos frequentemente essa prática de omitir os produtos em uso, talvez pelo receio da recriminação por parte do profissional em relação à automedicação.

Em conclusão, mostramos a possibilidade de alergia a produtos presentes em produtos de uso capilar como xampus e condicionadores. Alertamos que essa substância não está presente na atual bateria padrão brasileira. Reforçamos a necessidade de mostrar o risco da automedicação e de que somente um teste de contato ampliado e atualizado possibilitou o diagnóstico de uma forte sensibilidade a neomicina (inesperada) e de uma sensibilidade leve a cocoamidopropil betaína, mas relevante e geradora de toda a problemática.

\section{REFERÊNCIAS}

1. Nixon RL, Allnutt KJ, Diepgen TL. Contact Dermatitis. In: Adkinson NF. Middleton's Allergy Principle and Practice. $9^{\text {th }}$ ed, Philadelphia: Elsevier; 2020. p. 553-61.

2. Consenso "Dermatitis por Contacto". Sociedad Argentina de Dermatologia. Buenos Aires, 2015.

3. Rozas-Munoz E, Gamé D, Serra-Baldrich E. Dermatitis de contacto alérgica por regiones anatómicas. Claves diagnósticas. Actas Dermosifiliogr. 2018;109:485-507.

4. OjoEO,GowdaA, NedorostS.ScalpDermatitisinPatientsSensitized to Components of Hair Products. Dermatitis. 2019;30(4):264-7.

5. Jacob SE, Amini S. Cocamidopropyl betaine. Dermatitis. 2008;19(3):157-60.

6. Nettis $E$, Angelini G. Evaluation of the clinical relevance of a positive patch test reaction. In: Nettis E, Angelini G, Practical Guide to Patch Testing. $1^{\text {st }}$ ed, Cham: Springer Nature Switzerland; 2020. p. 33-40.

7. Pinto CC, Novell VS, Giménez-Arnau AM. Practical advice to correctly perform patch test. Curr Treat Options Allergy. 2019; 6:71-91.

8. Lachapelle JM. Baseline series of patch tests. In: Lachapelle JM, Maibach HI. Patch Testing and Prick Testing. $4^{\text {th }}$ ed, Cham: Springer Nature Switzerland; 2020. p. 85-104.

9. Grupo Brasileiro de Estudos em Dermatite de Contato. Estudo multicêntrico para elaboração de uma bateriapadrão brasileira de teste de contato. An Bras Dermatol. 2000;75(2):147-56.

10. DeKoven JG, Warshaw EM, Belsito DV, et al. NACDG patch test results. Dermatitis. 2017;28(1):33-46.

11. McFadden J, Puangpet P, Pongpairoj K, Thaiwat S, Xian LS. Neomycin. In: McFadden J. Common Contact Allergens: A Practical Guide to Detecting Contact Dermatitis. $1^{\text {st }}$ ed, John Wiley \& Sons; 2020. p. 287-91. 


\section{Conflito de interesse}

Não há.

Fonte de Financiamento Não há.

\section{Autor Correspondente:}

Paulo Eduardo Silva Belluco

belluco@outlook.com

Editor:

Prof. Dr. Marcelo Riberto

Recebido: 19/08/2020

Aprovado: 05/07/2021 